\title{
An Improved Cellular Automaton Model for Traffic Flow Under the Keep-Right-Except-To-Pass Rule Song Guo ${ }^{1, a}$

\author{
${ }^{1}$ School of Mathematical Science, Huaian Normal University
} \\ E-mail address: guosong77@sohu.com
}

\author{
Keywords: Cellular automaton model; NaSch model; Traffic flow; Keep-Right-Except-To-Pass \\ Rule; Lane-changing.
}

\begin{abstract}
Cellular automaton model has become an excellent tool for simulating real traffic flow, because of its efficient and fast performance in computer simulations. In this paper we investigate the two-lane cellular automaton traffic model considering lane-changing behavior under the Keep-Right-Except-To-Pass Rule based on a improved NaSch model. With the help of simulation results, we compare the traffic flow and the average velocities of the vehicles under the Keep-Right-Except-To-Pass Rule and under the no-pass rule or free-pass rule.
\end{abstract}

\section{Introduction}

In the recent years cellular automaton model had become an excellent tool for simulating real traffic flow, because of its efficiency in computer simulations. In 1992, Nagel and Schreckenberg proposed the well-known Nagel-Schareckenberg (NaSch) model [1]. Although it is a discrete model and very simple, the NaSch model can reproduce some real-traffic phenomena. Later, several extensions of the NaSch model were proposed, including single-lane models [2-7] and mutli-lane models [8-12].

In this paper we investigate the two-lane cellular automaton traffic model considering aggressive lane-changing behavior under the Keep-Right-Except-To-Pass Rule. Multi-lane freeways often employ a rule that requires drivers to drive in the right-most lane unless they are passing another vehicle, in which case they move one lane to the left, pass, and return to their former travel lane. The "aggressive" means drivers change lanes at once when the situations of speed and space guarantee them to pass the slower leading vehicle.

With the help of simulation results, we compare the traffic flow and the average velocities of the vehicles under the Keep-Right-Except-To-Pass Rule and the follow(no-pass) rule or free-pass rule. The free-pass rule is that when the drives are passing another vehicle, they won't return to their former travel lane. The simulations are based on the improved NaSch model. In order to simulate real-traffic, we lead into a slow-vehicle hypothesis: there are some vehicles with probability $\mathrm{p}_{\mathrm{s}}$ which maximum velocity is less than that of other vehicles. The hypothesis greatly reduces the average velocities of the vehicles, especially in light traffic and under no-pass rule. It will show the performance of the Keep-Right-Except -To-Pass Rule.

\section{Model}

\section{Model Assumptions}

We list the following assumptions throughout this paper:

(A_1) Ideal conditions: It refers to the good weather, and theroads are in good conditions.

(A_2) All the drivers obey the traffic rules, who are familiar with the traffic roads .

(A_3) Under the open boundary conditions, the generation of vehicles are subject to Poisson distribution, we mainly simulate the discrete state of vehicles, namely, various traffic characteristics when traffic density does not reach the critical value.

(A_4) The vehicles are random on the highway.

(A_5) Consider the highway as one-way two-lane road in this paper, and the right lane is slow 
lane(slow lane), the left lane for overtaking(fast lane).

(A_6) In passing process, the vehicles overtaken move at constant speeds.

Traffic flow is the number of motor vehicles in a section of the road in a unit time. To be specific, in a certain period of time, the number of vehicles passing some point in a section of the road. Passing sight distance refers to on the two lanes road, when the car passing the one immediately ahead, the shortest distance from leaving the original lane, to see the oncoming vehicle and after overtaking back to the original lane safely.

Basic model

We briefly recall the definition of the NaSch model[1]. The NaSch model is a discrete model for traffic flow. The road is divided into cells which can be either empty or occupied by the $n^{\text {th }}$ car with a velocity $v_{n}=0,1, \ldots, v_{n \max }$. The evolution are based on rules of NaSch model. Let $v_{n}(t)$ be the position of the $\mathrm{n}^{\text {th }}$ vehicle, $v_{n}(t)$ denote the velocity, $v_{n \text {,max }}$ denote the maximum velocity, $\operatorname{gap}_{n}(t)=v_{n+1}(t)-v_{n}(t)-1$ denote the distance between the $\mathrm{n}^{\text {th }} \quad$ vehicle and the nearest front one at time $t$. The vehicles move from the left to the right on a lane with periodic boundary conditions and the system update is performed in parallel for all cars according to the following four rules:

Acceleration: $v_{n} \rightarrow \min \left(v_{n}+1, v_{n, \max }\right)$; Deceleration: $v_{n} \rightarrow \min \left(v_{n}, g a p_{n}\right)$;

Randomization: $v_{n} \rightarrow \max \left(v_{n}-1,0\right)$ with probability $p_{n}$; Movement: $x_{n} \rightarrow x_{n}+v_{n}$.

Open boundary condition (cf. [2])\}

Under this question we expanded the width of the left boundary from one single cell to a minisystem of width $v_{\max }+1$. This is done to provide a proper insertion strategy allowing us to investigate the whole spectrum of possible system states. The allocation of the mini system (left boundary) has to be updated every time step before the vehicles of the complete system. The update procedure consist of two steps. If one cell of the mini system is occupied, it has to be emptied first. Then a vehicle with initial velocity $v_{\max }$ is inserted with probability $q_{\text {in }}$. Its position has to satisfy the following conditions: (i) The headway to the first car in main system is at least equal to the maximum velocity $v_{\max }$, and (ii)the distance to the main system has to be minimal, i.e., if no vehicle is present in the main system within the first $v_{\max }$ cells, the first cell of the boundary is occupied.

Under the open boundary conditions, the generation of vehicles are subject to Poisson distribution, we mainly simulate the discrete state of models, namely, various traffic characteristics when traffic density does not reach the critical value. Because of the number of vehicles lane is constantly changing, to characterize the dependency of the traffic flow on density, the quantity $\rho, \mathrm{V}$, $\mathrm{J}$ is defined as follows:

$$
\rho=\frac{N}{2 * L E N G T H}, V=\sum_{i=1}^{N} V_{i}(t), J=\rho V .
$$

Clearly, $\mathrm{V}$ is the mean velocity obtained by averaging over all cars of the system. According to the actual traffic conditions, we assume that the number of vehicles are subject to Poisson distribution on the driveway entrance in unit time, in which we only consider the vehicles on certain probability. The generation of vehicle are subject to Bernoulli distribution[2]. The time of generation of vehicle is at least $t$.

\section{Periodic Boundary Condition}

In this case we present results from system with periodic boundary conditions (thus simulating 
traffic on a circle "as in car races" but only on a single lane).For the car with $x_{n}>L$, we let $x_{n} \rightarrow x_{n}-L$, where $\mathrm{L}$ is the length of the circle. As the total number $\mathrm{N}$ of cars in the circle cannot change during the dynamics, we obtain a constant system density $\rho=N / L$. The traffic flow $\mathrm{J}$ is defined as follows:

$$
J=\rho \sum_{i=1}^{N} V_{i}(t) / N
$$

\section{Improved Model}

(1) Consider the road two-lane in parallel as one-dimensional discrete grid point chains, every lane is consisting of L grids, which is one-dimensional discrete grid point chains, and the distance between grids is 7.5 meter.

(2) The left lane is defined as Lane 1 (overtaking lane), the right lane is defined as Lane 2 (Lane). According to the given rule, vehicle in normal driving conditions only occupies lane 2, which can change lane. Lane 1 can only be used to overtake, which should be occupied as less as possible. The state of the $\mathrm{n}^{\text {th }}$ vehicle at time $\mathrm{t}$ is presented by its speed $v_{n}(t)$.

(3) State of every grid: empty( no vehicle) or occupied by a vehicle with speed of $v_{n}=0,1, \ldots, v_{n, \max }$ (Note that the maximum speed of $\mathrm{n}^{\text {th }}$ vehicle is $v_{n \text {, max }}$ ).

(4) Slow-vehicle hypothesis: there are some vehicles with probability $q_{s}$ which maximum velocity are less than other vehicles.

\section{Simulation}

There are two different types of vehicles, namely, fast vehicle and slow vehicle. Let the maximum velocity of slower vehicles is the maximum velocity of faster vehicles minus one. The maximum speed of the cars $v_{\max }=5$. Each cell corresponds to $7.5 \mathrm{~m}$ and a vehicle has a length of one cell. One time step corresponds to $1 \mathrm{~s}$. To be specific, we start with a random initial configuration of cars with density $\rho$ and velocity $\min \left(v_{\max }, g_{a p}\right)$ and begin the collection of data after the first $\mathrm{t}_{0}$ time steps, where we take $t_{0}=30000$. The line represents averages over 10000 time steps. To eliminate the random influence, we take twenty samples as the ensemble average.

\section{Light traffic}

Assume that the density $\rho<0.2$. In this case we carry out the simulations of the basic model and the improved model under Open boundary conditions. The vehicle insertion can be seen as sequence of Bernoulli trials[2], i.e., an insertion of a vehicle corresponds to a "success" $\mathrm{S}$ (probability $q_{i n}$ while a non-occupation corresponds to a "failure" F (probability $1-q_{i n}$ ). 


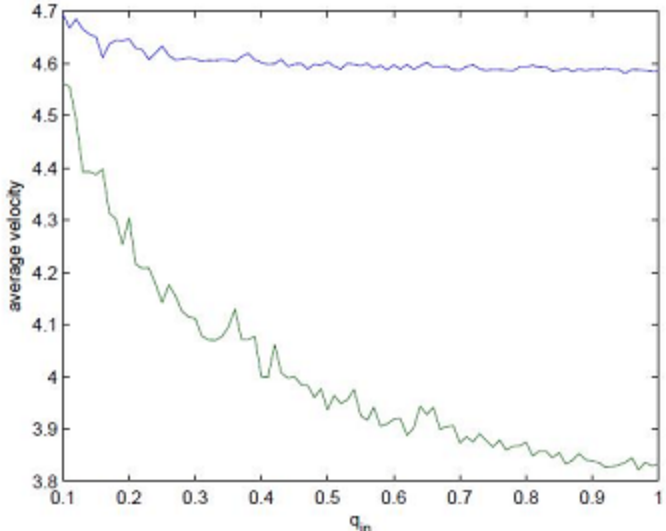

Figure 1: the ratio of slow vehicles $q=0.1$

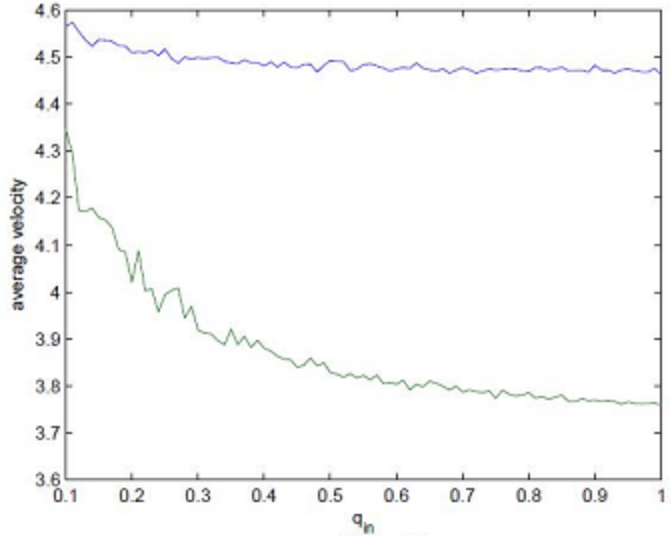

Figure 2: the ratio of slow vehicles $\mathrm{q} s=0.2$

Comparing the two average velocity curves in following Figure 1 and Figure 2, one can see the average velocities under Keep-Right-Except-To-Pass Rule clearly exceed the average velocities under the no-pass rule. The upper curves in the figures denote the the average velocities under Keep-Right-Except-To-Pass Rule and the lower curves denote the average velocities under the no-pass rule.

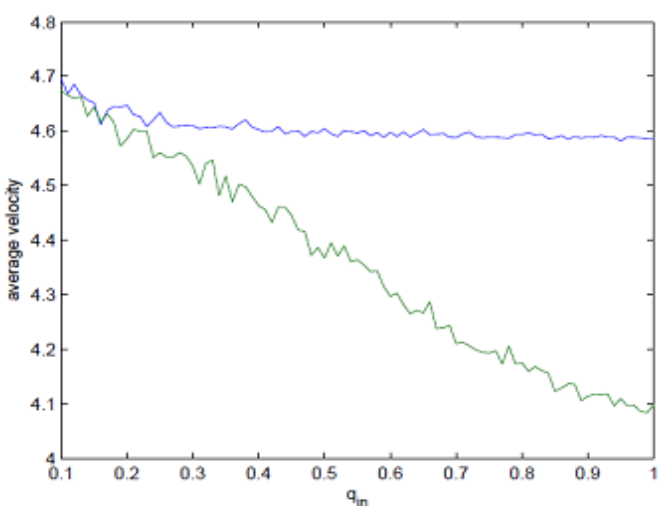

Figure 3: the ratio of slow vehicles $\mathrm{q} s=0.1$

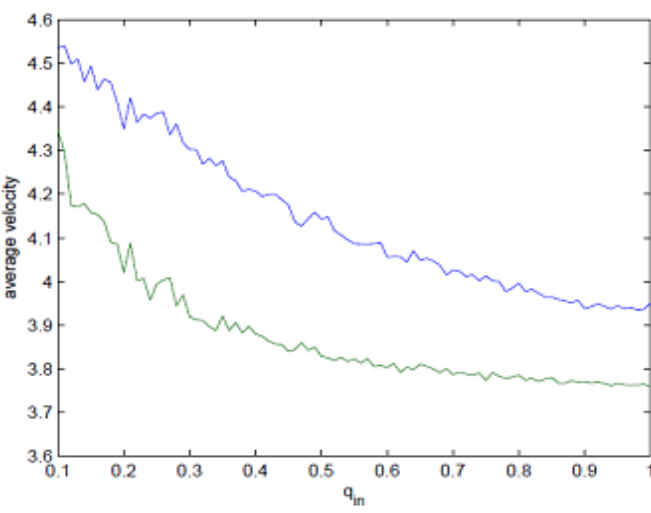

Figure 4: the ratio of slow vehicles $\mathrm{q} s=0.2$

Below we investigate the lane-changing under the free-pass rule: when the drives are passing another vehicle, they would not return to their former travel lane. In the Figure 3, The upper curves in the figures denote the the average velocities under Keep-Right-Except-To-Pass Rule and the lower curves denote the average velocities under the free-pass rule. We conclude that Keep-Right-Except-To-Pass Rule is better than free-pass rule and no-pass rule on raising the traffic flow and the velocities of the vehicles, and free-pass rule is better that no-pass rule.

\section{Heavy traffic}

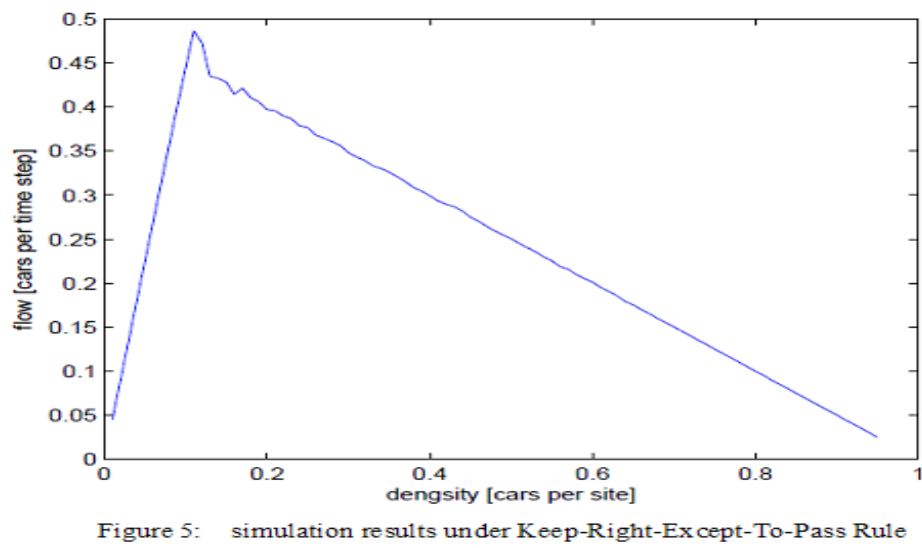

In this case we assume that $\rho \in[0,1]$. For a larger $\rho$, the average velocities of vehicles will be slower. The influences of slow-vehicle hypothesis become weakly. So we cancel the 
slow-vehicle hypothesis in the simulations, hence let $q_{s}=0$. We carry out the simulations of the improved model under the periodic boundary condition. Compared the flow curves in following figure with the figure in [1] , one can see the traffic flow under Keep-Right-Except-To -Pass Rule are more than the traffic flow under no-pass rule.

\section{Conclusion}

In this paper, we have investigated the aggressive lane-changing behavior under Keep-Right -Except-To-Pass Rule based on a improved cellular automaton model. The simulations announce that the average velocities and the traffic flow under Keep-Right-Except-To-Pass Rule are clearly larger than these quantities under the no-pass rule or the free-pass rule.

\section{Acknowledgements}

Fund Project: This author is supported by the National Natural Science Foundation (11471138) of China and Natural Science Research Project of Ordinary Universities in Jiangsu Province (13KJB110001),P.R.China.

\section{References}

[1] K. Nagel, M. Schreckenberg, A cellular automaton model for freeway traffic\}, J. Phys. I(France) 2 (1992), 2221-2229.

[2] R. Barlovic, T.Huisinga,A. Schadschneider and M. Schreckenberg, Open boundaries in a cellular automaton model model for traffic flow with metastable states,Physical Reviews E 66(2002),046113.

[3] H.Emmerich,E.Rank, An improved cellular automaton model for traffic flow simulation,Phusica A 234(1997) 676-686.

[4] M.Cremer and J.Ludwing, A fast simulation model for traffic flow on the basis of Boolean operation,Mathematics and Computers in Simulation(1986),28:297-303.

[5] Wei Bai,Cun-jun Li, Overtaking model based on different limiting speed, Journal of Transportation System Engineering and Information Technology,2013,13(2).

[6] Jiu-Chun Gu,Yan Chen, Hai-Long Wang, Quan-Zhong Zhang, Study on cellular automaton model consider freight vehicles, Computer Engineering and Applications 46(7)(2010),236-238.

[7] Xin-Gang Li, Bin Jia, Zi-You Gao, Rui Jiang, A realistic two-lane cellular automata traffic model considering aggressive lane-changing behavior of fast vehicle ,Physica A 367(2006), 2221-2229.

[8] Da-Yan Wu,Hui-Li Tan,Ling-Jiang Kong,Mu-Ren Liu, Study on a three-lane cellular automata traffic flow model, Journal of systems engneering , August(2005),393-397.

[9] Peng Lin,Hui-Li Tan,Ling-Jiang Kong,Mu-Ren Liu, A study of coupling effect in cellular automata model of traffic flow for two-lane with open boundary conditions, Acta Physica Sinica, December(2003),3007-3012.

[10] Tie-qiao Tang,Hai-Jun Huang,Chao-Qun Mei, An improved overtaking model, Journal of Transportation Systems Engineering and Information Technology(2005),5(4),23-25.

[11] Fu-wei Wu, Jia-he Qin,Chao-wei Ren,Zeng-liang Niu,Jun-lei Zhang, Simulation of automatic overtaking control algorithm for intelligent vehicle on highway,Computer Engineering and Design,2013,34(7).

[12] Zuo- Jin Zhu, Qing-Song Wu, Rui Jiang, et al. Numerical study on traffic flow with single parameter state equation[ J] . J. Trans. Engineering , 2002, 128( 2) : 167- 172. 